\title{
Blue-on-Green Flash Induces Maximal Photopic Negative Response and Oscillatory Potential and Serves as a Diagnostic Marker for Glaucoma in Rat Retina
}

\author{
Su Jin Park ${ }^{1,2}$, Sun Sook Paik ${ }^{1,2 *}$, Ji-Yeon Lee ${ }^{1}$, Su-Ja Oh ${ }^{1}$ and In-Beom Kim ${ }^{1,2,3 *}$ \\ ${ }^{1}$ Department of Anatomy, College of Medicine, The Catholic University of Korea, Seoul 06591, \\ ${ }^{2}$ Catholic Neuroscience Institute, College of Medicine, The Catholic University of Korea, Seoul 06591, \\ ${ }^{3}$ Catholic Institute for Applied Anatomy, College of Medicine, The Catholic University of Korea, Seoul 06591, Korea
}

\begin{abstract}
The purpose of this study was to investigate the application of various electroretinography (ERG) to the diagnosis of inner retinal dysfunction induced by mild intraocular pressure (IOP) elevation in a rat glaucoma model. For inner retinal function measurements, available photopic ERG protocols were applied under various light conditions including monochromatic combinations, which complement conventional scotopic ERG. Three episcleral veins in the right eyes of Sprague-Dawley rats were cauterized to induce an experimental model of glaucoma, leading to mild IOP elevation. ERG responses were measured before surgery and at 1, 2,4 , and 8 weeks after cauterization. We first confirmed that the amplitude reduction in the standard photopic b-wave was almost comparable to the amplitudes of scotopic a- and b-waves in glaucomatous eyes over time. We have implemented additional photopic ERG protocols under different stimulus conditions, which consisted of a longer duration and different monochromatic combinations. Such a change in the stimulations resulted in more pronounced differences in response between the two groups. Especially in normal animals, blue stimulation on a green background produced the largest b-wave and photopic negative response (PhNR) amplitudes and caused more pronounced oscillatory potential (OP) wavelets (individual components). In glaucomatous eyes, blue stimulation on a green background significantly reduced PhNR amplitudes and abolished the robust OP components. These results, by providing the usefulness of blue on green combination, suggest the applicable photopic ERG protocol that complements the conventional ERG methods of accessing the progression of glaucomatous damage in the rat retina.
\end{abstract}

Key words: Electroretinography, Photopic negative response, Oscillatory potential, Glaucoma, Rat retina

Received April 26,2018, Revised May 23, 2018,

Accepted May 28, 2018

* To whom correspondence should be addressed.

Sun Sook Paik

TEL: 82-2-2258-7268, FAX: 82-2-536-3110

e-mail: paikss100@hanmail.net

In-Beom Kim

TEL: 82-2-2258-7263, FAX: 82-2-536-3110

e-mail: ibkimmd@catholic.ac.kr

\section{INTRODUCTION}

Glaucoma is an optic neuropathy characterized by progressive degeneration of retinal ganglion cells (RGCs), visual field loss, and occasionally intraocular pressure (IOP) elevation $[1,2]$. The slow RGC damage observed in glaucoma is also seen in many other optic neuropathies and retinopathies in acute IOP elevation induced
Copyright $\odot$ Experimental Neurobiology 2018. www.enjournal.org
This is an Open Access article distributed under the terms of the Creative Commons Attribution Non-Commercial License (http://creativecommons.org/licenses/by-nc/4.0) which permits unrestricted non-commercial use, distribution, and reproduction in any medium, provided the original work is properly cited. 
models. Examples include optic nerve transection (ONT) and central vein occlusion in an ischemia model. Therefore, it is necessary to exploit the advantages of a moderate IOP elevation model to study inner retinal dysfunction that can only be observed in glaucoma $[3,4]$.

One of the most convenient and noninvasive techniques to track the functional state of the glaucomatous retina is electroretinography (ERG), which provides direct evaluations of retinal cell function and the physiological mechanism $[1,5]$. Under the fullfield flash condition, both scotopic and photopic ERG parameters minimally reflect inner retinal function [6] with considerable variability between subjects. Even in a rat model after optic nerve transection, one report demonstrated a reduction in the scotopic a-wave amplitude [7]. Additionally, studies in human subjects with glaucoma have proven that there are relatively few changes in basic scotopic ERG responses [8] but more significant alterations in oscillatory potentials (OPs) and pattern ERG amplitudes [9]. As described above, despite the fact that third-order retinal neurons are known to affect ERG, their contribution to the standard scotopic ERG is more difficult to measure. Responses to moderately bright flashes under light-adapted conditions have greater utility in this regard [10].

There have been a number of studies showing alternative ERG applications that reveal inner retinal function. The slow photopic negative response (PhNR) following the b-wave on longer photopic ERG is one such method [11]. The PhNR is probably generated as a consequence of the $\mathrm{Na}^{+}$-dependent spiking activity of retinal ganglion cells (RGCs) [12]. Another ERG component related to inner retinal function is OPs, which are the fast wavelets on the ascending edge of the b-wave on flash ERG. They are thought to be generated by bipolar and amacrine cells localized in the inner nuclear layer (INL) in response to inhibitory feedback involving mainly amacrine cells. In a recent study, the analysis of OPs was thought to be a potential indicator for evaluating retinal dysfunction related to optic nerve disease [13]. Interestingly, most OPs are known to be more visible under scotopic conditions but rarely have been studied under photopic conditions we found new waveforms in such attempts [14].

In the present study, we employed prolonged light stimulation (200 ms) using various color combinations for the stimuli and backgrounds to isolate the maximal cone-driven responses under rod saturation and to evoke the maximal responses of two photopic components: the PhNR and OPs. Through validation of the usefulness of the blue light stimulus on green background, we suggest the applicable photopic ERG protocol that complements the conventional ERG methods of accessing the progression of glaucomatous damage in the rat retina.

\section{MATERIALS AND METHODS}

\section{Animals}

Male Sprague-Dawley rats (8 weeks old, 250 300 g) (from Orient Bio Co., Seongnam-si, Gyeonggi-do, Korea) were used in this study. A total of 60 rats were used in 5 groups of glaucomatous eyes ( $n=6$ per group) and 5 groups of age-matched controls $(n=6$ per group). All rats were handled in accordance with regulations established by the Catholic Ethics Committee of the Catholic University of Korea, Seoul, which conform to the National Institutes of Health (NIH) Guide for the Care and Use of Laboratory Animals (NIH Publications, no. 80-23, revised 1996). Rats were kept alive for up to $1,2,4$, and 8 weeks in each group after cauterization and sacrificed by an overdose of anesthetics.

\section{Glaucoma model}

Episcleral vein cauterization, also known as the Shareef model, was employed to induce experimental glaucoma in rat eyes. Histological findings and its analysis for this glaucoma model was described in our previous studies $[15,16]$. Rats were anesthetized with intraperitoneal injections of a mixture of tiletamine plus zolazepam hydrochloride $(50 \mathrm{mg} / \mathrm{kg})$ and xylazine $(10 \mathrm{mg} / \mathrm{kg})$. Topical proparacaine $(0.5 \%)$ was used for corneal anesthesia and 1 drop was applied to the right eye before surgery. A small incision was made in the conjunctiva of the right eye. Generally, three episcleral veins were cauterized using a hand-held cautery device (Change-A-Tip Deluxe Low-temp Cautery Kit, Bovie Medical Corporation, Purchase, NY, USA) (Fig. 1A). To prevent heat damage to the surrounding sclera, a small piece of wood was placed under the vein to separate it from the surrounding tissue.

\section{IOP measurement}

IOP was measured using a TonoLab tonometer (Icare ${ }^{\circledR}$, OY, Helsinki, Finland). Tonometers are based on the rebound measurement principle in which a very lightweight probe is used to make momentary contact with the cornea. Animals were kept as calm as possible to minimize the effect of stress on IOP readings. Ten readings were taken and averaged to give 1 measurement. Initial IOP measurements were performed 1 day before cauterization to determine the baseline IOP. After cauterization, IOP measurements were repeated at $1,2,4$, and 8 weeks.

\section{ERG recordings}

Full-field ERG was recorded simultaneously from both eyes to assess functional alterations. Animals were kept in a dark room for $16 \mathrm{~h}$ before the ERG recording. All recording procedures were performed under dim red light $(\lambda>600 \mathrm{~nm})$. Simultaneous recording 
A

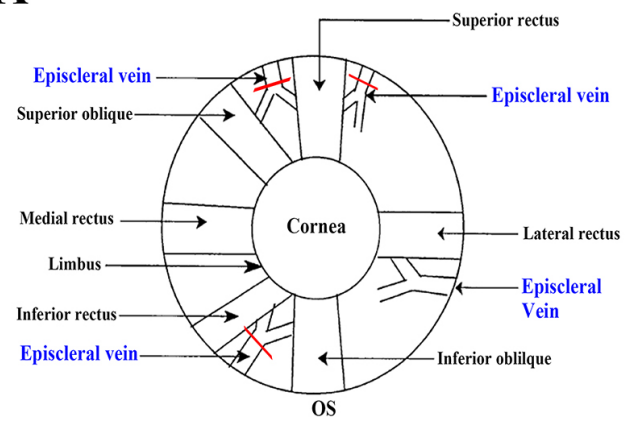

C
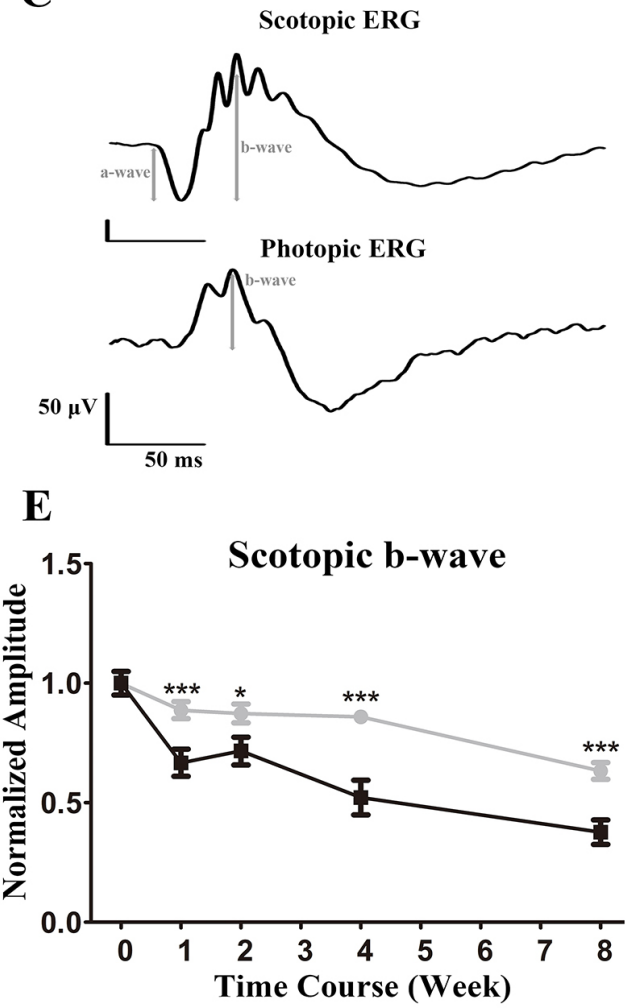

B

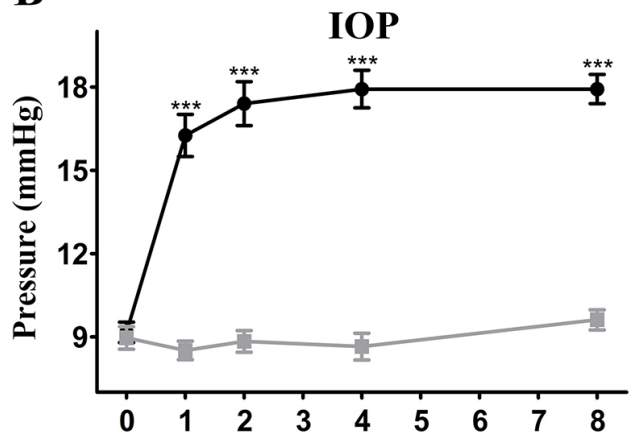

D

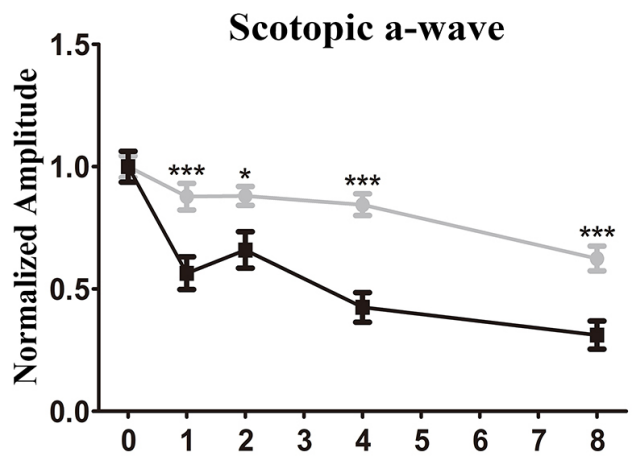

F

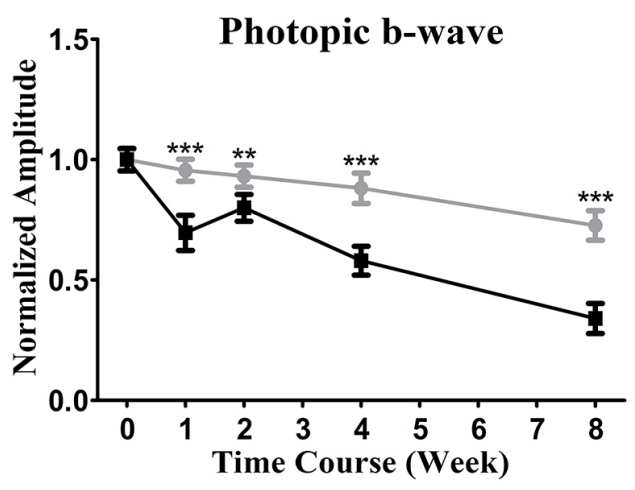

Fig. 1. The progression of intraocular pressure (IOP) elevation and electroretinography (ERG) amplitudes recorded from brief white stimuli after episcleral vein cauterization. (A) Diagram of the episcleral vein. (B) IOP changes from the age-matched control group and the glaucomatous group are plotted over time. The results are expressed as the mean IOP \pm the standard error of the mean (SEM) ( $\mathrm{n}=6$ in the control group and in each time group). (C) Representative waveforms of scotopic, photopic ERG in normal rat. (D-E) Scotopic ERG data are shown as the normalized amplitudes of a- and bwaves. (F) Photopic ERG is represented as normalized b-wave amplitudes over time. The results are expressed as the mean normalized amplitude \pm SEM ( $\mathrm{n}=6$ in the control group and in each time group). Significant differences at 1,2,4, and 8 weeks denoted as asterisk $\left(^{*}\right)$ are from control vs. glaucoma groups. ${ }^{*} \mathrm{p}<0.05,{ }^{* *} \mathrm{p}<0.01,{ }^{* * *} \mathrm{p}<0.001$.

allowed ERG to be obtained from the control and cauterized eyes under identical states of anesthesia. Hydroxypropyl methylcellulose gel was applied to the corneas and covered with gold ring contact electrodes. A ground electrode and a reference electrode were placed subcutaneously in the tail and ear, respectively. Brief white flash stimuli were delivered via a Ganzfeld stimulator (UTAS-3000; LKC Technologies, Gaithersburg, MD, USA). Signals were amplified and filtered through a digital band-pass filter ranging from 5
$\mathrm{Hz}$ to $1 \mathrm{kHz}$ to yield a- and b-waves. Scotopic ERG was measured at $0.99 \mathrm{~cd} \cdot \mathrm{s} / \mathrm{m}^{2}$. Averaged responses were obtained from multiple stimulus trials that were separated by a 15- second inter-stimulus interval. The amplitude of the scotopic a-wave was measured from the baseline to the negative peak. The amplitude of the b-wave was measured from the trough of the a-wave to the following response peak. Photopic ERG was recorded on a white rod-suppressing background of $30 \mathrm{~cd} / \mathrm{m}^{2}$ after light adaptation for 15 minutes. 
Light-adapted flash responses were measured at $6.28 \mathrm{~cd} \cdot \mathrm{s} / \mathrm{m}^{2}$. Each measurement was an average of 5 responses obtained within a 5 second inter-stimulus interval. Amplitudes of photopic b-waves were measured from the baseline to the positive peak.

For measurements of various photopic components such as the PhNR and OPs that can assist in a comprehensive analysis of retinal function using scotopic ERG, we applied prolonged, monochromatic, full-field stimuli and steady background illumination to saturate the rods. The stimuli were blue flashes $\left(6.28 \mathrm{~cd} / \mathrm{m}^{2}\right)$ presented via a Ganzfeld integrating sphere after 15 minutes of light adaptation on a blue $(450 \mathrm{~nm})$ and green $(560 \mathrm{~nm})$ background $\left(10 \mathrm{~cd} / \mathrm{m}^{2}\right)$ for a long duration $(200 \mathrm{~ms})$. Each recording was an average of 10 sweeps with an inter-stimulus interval of $0.4 \mathrm{~s}$. Signals were band-pass filtered between $10 \mathrm{~Hz}$ to $1 \mathrm{kHz}$. The PhNR amplitude was measured from the baseline to the trough following the b-wave.

\section{Data acquisition and statistical analysis}

The data analysis was performed with Origin 8 (Origin Lab, Northampton, MA, USA) and statistical analyses were performed using Student's $t$ tests. Data are presented as the mean \pm the standard error of the mean (SEM). Differences were considered statistically significant at $\mathrm{p}<0.05$.

\section{RESULTS AND DISCUSSION}

Episcleral vein cauterization causes glaucoma by increasing IOP. IOP was measured before cauterization and at 1,2, 4, and 8 weeks after cauterization and was also recorded in the age-matched control groups at each time point. The IOP was $9.16 \pm 0.37 \mathrm{mmHg}$ $($ mean \pm SEM) in normal eyes before cauterization (Fig. 1B). The IOP rapidly increased to $16.26 \pm 0.76 \mathrm{mmHg}$ at 1 week following cauterization and an elevated IOP was maintained for 8 weeks. In the control group, the IOP was maintained at an average of 8.65 $\mathrm{mmHg}$ from 0 to 4 weeks and slightly increased at 8 weeks to $9.61 \pm 0.36 \mathrm{mmHg}(\mathrm{p}<0.001)$.

Under brief scotopic and photopic conditions, full-field ERG recordings were performed to evaluate the progression of glaucoma. We tested whether standard ERG components such as a- and bwave changes reflected inner retinal function (Fig. 1C). The time courses of the scotopic and photopic ERG amplitudes are shown for comparisons between control and glaucomatous eyes (Fig. 1D F). Scotopic a- and b-waves and photopic b-waves were significantly reduced at 1 week, slightly recovered at 2 weeks, and then decreased again until 8 weeks $(\mathrm{p}<0.05)$. The overall tendencies of decline were similar between the scotopic and photopic ERG responses, which may be related to the loss of function in the rela- tively outer retina rather than inner retina. Therefore, additional ERG methods are essential for measuring the retinal dysfunction in the rat glaucoma model.

We attempted to investigate photopic ERG and especially focused on 2 photopic ERG parameters, the PhNR and OPs, by employing prolonged light in combination with various monochromatic backgrounds and test stimuli in rat eyes. In normal rats (8 weeks old), the PhNR was measured under green-on-blue $(\mathrm{G} / \mathrm{B})$, blue-on-blue $(B / B)$, and blue-on-green $(B / G)$ conditions, as well as under the red-on-blue (R/B) condition used in humans and macaque monkeys. Fig. 2A shows representative photopic ERG traces for each color combination condition. The amplitudes of the PhNR under R/B, G/B, B/B, and B/G conditions were $10.28 \pm 2.6$ $\mu \mathrm{V}, 30.2 \pm 3.2 \mu \mathrm{V}, 94.67 \pm 4.5 \mu \mathrm{V}$, and $133.74 \pm 5.3 \mu \mathrm{V}$, respectively (Fig. $2 \mathrm{~B})$. In addition, individual OPs were prominently observed on the b-waves (Fig. 2A). We quantified OP activities by counting the number of obvious distinct OPs. Such quantification is uncommon, but the addition of an OP component under the different light conditions is considered to be a very important finding, as elucidated further in the Discussion. The number of OPs under each condition was counted and averaged (Fig. 2C). The numbers of OPs under $\mathrm{R} / \mathrm{B}, \mathrm{G} / \mathrm{B}, \mathrm{B} / \mathrm{B}$, and $\mathrm{B} / \mathrm{G}$ conditions were $0.8 \pm 0.3$, $1.7 \pm 0.2,2.3 \pm 0.3$, and $3.8 \pm 0.1$, respectively. Both PhNR amplitudes and OPs were significantly higher under the $\mathrm{B} / \mathrm{G}$ condition than under other flash conditions $(\mathrm{p}<0.05)$. These results indicate that the $\mathrm{PhNR}$ and OPs under the $\mathrm{B} / \mathrm{G}$ condition are good parameters to reveal inner retinal function, leading us to the working hypothesis that these photopic ERG components can be applied to monitor inner retinal dysfunction in a rat glaucoma model.

Next, we applied these two photopic ERG components to glaucomatous rat eyes. The PhNR traces were arranged according to the time points in the glaucoma model (Fig. 2D). The amplitude changes in the PhNR were dramatic as glaucoma progressed compared with those of the controls. In age-matched control rat eyes, PhNR amplitudes were relatively maintained but slightly decreased under the $\mathrm{B} / \mathrm{G}$ condition $(133.74 \pm 5.3 \mu \mathrm{V}$ vs. $103.07 \pm 5.1$ $\mu \mathrm{V})$. On the other hand, PhNR amplitudes in experimental glaucomatous eyes were drastically decreased. After the induction of experimental glaucoma by cauterization, the amplitudes were $57.9 \pm 13.4,76.4 \pm 5.0,52.4 \pm 7.2$, and $28.5 \pm 6.1 \mu \mathrm{V}$ for 1,2 , 4 , and 8 weeks, respectively. The PhNR amplitudes are presented as plotted normalized data (Fig. 2E) $(\mathrm{p}<0.05)$. In age-matched control eyes, photopic ERGs showed 3 to 4 large and distinct OP wavelets. In the age-matched control group, the number of OPs remained relatively constant $(3.9 \pm 0.1$ vs. $3.3 \pm 0.3)$. On the other hand, in the glaucoma group, it decreased to $2.25 \pm 0.3$ at 1 week after cauterization, rebounded to $3.25 \pm 0.2$ at 2 weeks, decreased to $2.38 \pm 0.3$ at 
A

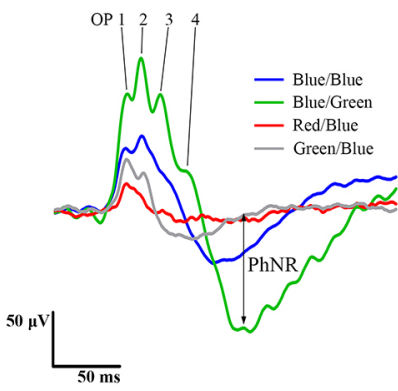

D

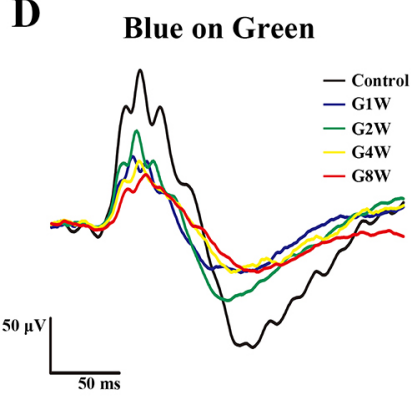

B

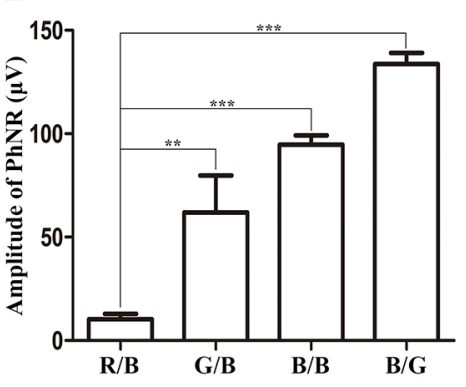

E

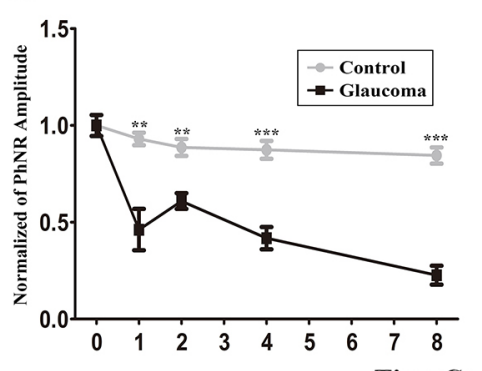

C

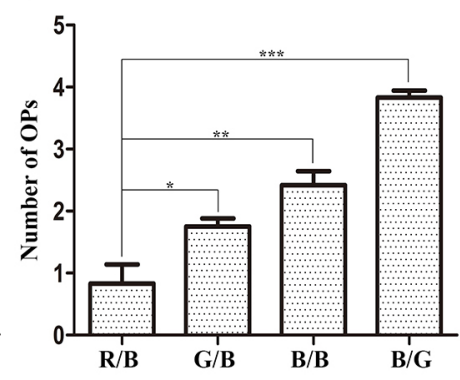

F

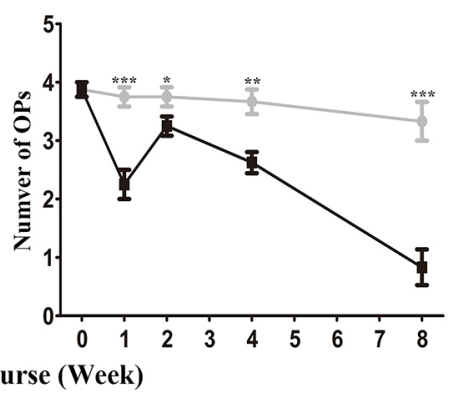

Fig. 2. An associative analysis of prolonged photopic electroretinography (ERG) responses including all components using light stimuli. (A) Representative traces of each prolonged photopic ERG response from a normal rat eye are shown for different color combinations. (B) In normal rats, differences in the photopic negative response (PhNR) amplitude are compared according to color combination conditions. (C) Under the same longer photopic flash that elicited the PhNR, oscillatory potentials (OPs) are measured by the number of prominent wavelets and compared according to the color combinations. (D) The responses from prolonged photopic ERG are shown as a trace from 0 to 8 weeks after cauterization. The data values are presented as the mean ( $\mathrm{n}=6$ in the control group and in each time group). (E) The change in PhNR amplitude over time is presented as normalized data. The results are expressed as the mean amplitude \pm the standard error of the mean (SEM) ( $\mathrm{n}=6$ in the control group and in each time group). (F) The number of OP wavelets is analyzed over time under blue stimulation/green background $(\mathrm{B} / \mathrm{G})$ conditions. The data values are presented as the mean \pm SEM $(\mathrm{n}=6$ in the control group and in each time group). ${ }^{*} \mathrm{p}<0.05,{ }^{* *} \mathrm{p}<0.01,{ }^{* * *} \mathrm{p}<0.001$.

4 weeks, and then decreased again to $0.83 \pm 0.3$ at 8 weeks. Thus, at 8 weeks, prominent OPs were rarely observed (Fig. 2F) ( $\mathrm{p}<0.05)$. Therefore, the $\mathrm{B} / \mathrm{G}$ combination facilitated PhNR and OPs activities in normal rat eyes and made the change in the number of OP wavelets over time more apparent.

In this study, in order to assess the progression of inner retinal dysfunction in a rat glaucoma model, along with the conventional scotopic and photopic flash ERGs, longer photopic flash ERGs (eg. photopic negative response $[\mathrm{PhNR}]$ ) were employed by changing the combination of blue and green flash. Through validation of the usefulness of the blue light stimulus on green background, we suggest the applicable photopic ERG protocol that complements the conventional ERG methods of accessing the progression of glaucomatous damage in the rat retina.

Scotopic ERG has been utilized in a more important way to measure retinal function in a rat model, because the rat is a roddominant animal [17]. As an alternative to conventional ERG, assessment of retinal function in dark-adapted conditions such as scotopic threshold response (STR) has become more important in rat models $[18,19]$. Nonetheless, Bui et al. [10] suggested that a bright light flash under light-adapted conditions might have greater utility in evaluating the function of third-order neurons. In our experiment, as glaucoma progressed, changes in the b-waves of photopic ERG responses were almost comparable to the changes in both the a- and b-waves of the scotopic responses. Bayer et al. [1] showed that photopic b-waves reflected inner retinal dysfunction in the same glaucoma model as ours. Another study demonstrated that the cone response contributed substantially to the b-wave at an increased light level in the rat retina [20]. With the same context, our study suggests that photopic ERG might be an important way to measure retinal function changes in rat glaucoma models.

Based on photopic ERG, we tried color combinations and a long duration to find alternative photopic ERG components that reflect inner retinal function. The photopic component observed under such conditions is the PhNR; previous studies on the PhNR in rodents were conducted under $\mathrm{R} / \mathrm{B}$ or white conditions $[6,21$ 23]. Unlike their attempts, we changed the conditions to consist of different combinations of monochromic test stimuli on a back- 
ground illumination $(\mathrm{R} / \mathrm{B}, \mathrm{G} / \mathrm{B}, \mathrm{B} / \mathrm{B}$, and $\mathrm{B} / \mathrm{G})$ for a prolonged duration (200 ms). As a result, the $\mathrm{B} / \mathrm{G}$ condition yielded the largest $\mathrm{PhNR}$ amplitude. The $\mathrm{B} / \mathrm{G}$ condition is more likely to cause an effect, considering that rat rods are sensitive to short wavelengths, while the majority of cones are sensitive to medium-to-long wavelengths with a small proportion having short-wavelength sensitivity [24]. However, the rat ERG has 3 spectral peaks, so a fullfield green background may play a role as a stimulus for most rat cones rather than as a saturator for rods. Additionally, cone-driven RGC responses may be related to incremental changes in PhNR amplitudes. The stimulus condition for the PhNR in the present study supports the idea that a monochromatic full-field test flash may evoke a more obvious PhNR than broadband stimuli because it provides less opportunity for inhibitory center-surrounding interactions in the responses of color-opponent RGCs [25]. In addition, monochromatic flash with $\mathrm{B} /$ yellow flash based on the spectral sensitivity of the human cone photoreceptors may be effectively applied to glaucoma monitoring in human [26], as B/G flash induces maximal PhNR in this study.

Another important finding related to inner retinal function is that the $\mathrm{B} / \mathrm{G}$ condition produced more prominent $\mathrm{b}$-waves and OPs in the rats. Although there are a number of studies in which OPs were associated with inner retinal function, little is known about the contribution of photopic OPs to the light-adapted rat retina. Under the light condition, the numbers and amplitudes of OPs were significantly increased, and in particular, the number of $\mathrm{OP}$ wavelets changed prominently as glaucoma progressed. Based on a study showing that the number of components in OPs was highly correlated with retinal function originating from various inner regions [27], generation of a new OP component would be a very interesting and important finding. Considering that the latter OP component is generally considered to be closer to the proximal region [27], it may be evidence that the newly appearing OP component further reflects inner retinal function. This is the first result demonstrating photopic condition-induced OP components that reflect gradual damage to inner retinal function in a rat glaucoma model.

The glaucoma model applied in this study is characterized by mild elevation of IOP ( $20 \mathrm{mmHg})$. One document of rat glaucoma has proven that during acute IOP elevation, functional changes progress from proximal to distal regions [14]. They have also addressed that nonspecific functional changes were observed in acute IOP above $50 \mathrm{mmHg}$, suggesting that IOP should be maintained below this level in experimental glaucoma models if selective RGC injury is to be sought. Although our glaucoma model is basically different from an acute model, we can infer that the relatively long duration of mild elevation of IOP would have a specific effect on inner retinal function. Thus, our experiments suggest that photopic sensitivity may be relatively high in models with mild elevation of IOP, in contrast to Buiss group study on photopic sensitivity in an acute IOP model [14], and raise the possibility that photopic ERG can detect progressive inner retinal dysfunction in glaucomatous rats more sensitively than full-field scotopic ERG, which is difficult to measure changes in inner retinal function.

Although we find and provide the best color combination leading the maximal photopic ERG responses reflecting inner retinal function in normal and glaucomatous rat retinas, we need to mention that in rodent glaucoma models, their usefulness is compared to that of scotopic ERG responses, such as scotopic OP and STR, which are thought to be generated by feedback from amacrine cells coupled to RGCs in rodents under the dark-adapted condition $[28,29]$. That is, further study will be needed to examine whether significant changes in STR or scotopic OP can be detected even in mild elevation of IOP condition ( $20 \mathrm{mmHg})$. It may contribute to more accurate assessment of inner retinal function changes in the rat glaucoma model.

\section{ACKNOWLEDGEMENTS}

This work was supported by National Research Foundation of Korea (NRF) funded by the Ministry of Science and ICT (NRF2017R1A2B2005309) and Seoul St. Mary’s Hospital (R\&D 2016 program).

\section{REFERENCES}

1. Bayer AU, Danias J, Brodie S, Maag KP, Chen B, Shen F, Podos SM, Mittag TW (2001) Electroretinographic abnormalities in a rat glaucoma model with chronic elevated intraocular pressure. Exp Eye Res 72:667-677.

2. Hernandez M, Rodriguez FD, Sharma SC, Vecino E (2009) Immunohistochemical changes in rat retinas at various time periods of elevated intraocular pressure. Mol Vis 15:26962709.

3. Kiszkielis M, Lubiński W, Penkala K (2012) The photopic negative response as a promising diagnostic tool in glaucoma. A review. Klin Oczna 114:138-142.

4. Wilsey LJ, Fortune B (2016) Electroretinography in glaucoma diagnosis. Curr Opin Ophthalmol 27:118-124.

5. Bui BV, Fortune B (2004) Ganglion cell contributions to the rat full-field electroretinogram. J Physiol 555:153-173.

6. Smith BJ, Wang X, Chauhan BC, Côté PD, Tremblay F (2014) Contribution of retinal ganglion cells to the mouse electroretinogram. Doc Ophthalmol 128:155-168. 
7. Alarcón-Martínez L, de la Villa P, Avilés-Trigueros M, Blanco R, Villegas-Pérez MP, Vidal-Sanz M (2009) Short and long term axotomy-induced ERG changes in albino and pigmented rats. Mol Vis 15:2373-2383.

8. Fazio DT, Heckenlively JR, Martin DA, Christensen RE (1986) The electroretinogram in advanced open-angle glaucoma. Doc Ophthalmol 63:45-54.

9. Vaegan SL, Graham SL, Goldberg I, Millar TJ (1991) Selective reduction of oscillatory potentials and pattern electroretinograms after retinal ganglion cell damage by disease in humans or by kainic acid toxicity in cats. Doc Ophthalmol 77:237253.

10. Bui BV, Fortune B, Cull G, Wang L, Cioffi GA (2003) Baseline characteristics of the transient pattern electroretinogram in non-human primates: inter-ocular and inter-session variability. Exp Eye Res 77:555-566.

11. Bach M, Poloschek CM (2013) Electrophysiology and glaucoma: current status and future challenges. Cell Tissue Res 353:287-296.

12. Viswanathan S, Frishman LJ, Robson JG, Harwerth RS, Smith EL 3rd (1999) The photopic negative response of the macaque electroretinogram: reduction by experimental glaucoma. Invest Ophthalmol Vis Sci 40:1124-1136.

13. Post M, Goslawski W, Modrzejewska M, Wielusinski M, Kazmierczak J, Lubinski W (2015) Electrophysiological function of the retina and optic nerve in patients with atrial fibrillation. Doc Ophthalmol 131:53-62.

14. Bui BV, Edmunds B, Cioffi GA, Fortune B (2005) The gradient of retinal functional changes during acute intraocular pressure elevation. Invest Ophthalmol Vis Sci 46:202-213.

15. Park CK, Cha J, Park SC, Lee PY, Kim JH, Kim HS, Kim SA, Kim IB, Chun MH (2009) Differential expression of two glutamate transporters, GLAST and GLT-1, in an experimental rat model of glaucoma. Exp Brain Res 197:101-109.

16. Park HY, Kim JH, Park CK (2014) Alterations of the synapse of the inner retinal layers after chronic intraocular pressure elevation in glaucoma animal model. Mol Brain 7:53.

17. Szél A, Röhlich P (1992) Two cone types of rat retina detected by anti-visual pigment antibodies. Exp Eye Res 55:47-52.

18. Choh V, Gurdita A, Tan B, Prasad RC, Bizheva K, Joos KM
(2016) Short-term moderately elevated intraocular pressure is associated with elevated scotopic electroretinogram responses. Invest Ophthalmol Vis Sci 57:2140-2151.

19. Fortune B, Bui BV, Morrison JC, Johnson EC, Dong J, Cepurna WO, Jia L, Barber S, Cioffi GA (2004) Selective ganglion cell functional loss in rats with experimental glaucoma. Invest Ophthalmol Vis Sci 45:1854-1862.

20. Nixon PJ, Bui BV, Armitage JA, Vingrys AJ (2001) The contribution of cone responses to rat electroretinograms. Clin Exp Ophthalmol 29:193-196.

21. Chrysostomou V, Crowston JG (2013) The photopic negative response of the mouse electroretinogram: reduction by acute elevation of intraocular pressure. Invest Ophthalmol Vis Sci 54:4691-4697.

22. Li B, Barnes GE, Holt WF (2005) The decline of the photopic negative response $(\mathrm{PhNR})$ in the rat after optic nerve transection. Doc Ophthalmol 111:23-31.

23. Yun H, Lathrop KL, Yang E, Sun M, Kagemann L, Fu V, Stolz DB, Schuman JS, Du Y (2014) A laser-induced mouse model with long-term intraocular pressure elevation. PLoS One 9:e107446.

24. Cicerone CM (1976) Cones survive rods in the light-damaged eye of the albino rat. Science 194:1183-1185.

25. Viswanathan S, Frishman LJ, Robson JG, Walters JW (2001) The photopic negative response of the flash electroretinogram in primary open angle glaucoma. Invest Ophthalmol Vis Sci 42:514-522

26. Wakili N, Horn FK, Jünemann AG, Nguyen NX, Mardin CY, Korth M, Kremers J (2008) The photopic negative response of the blue-on-yellow flash-electroretinogram in glaucomas and normal subjects. Doc Ophthalmol 117:147-154.

27. Wachtmeister L (1998) Oscillatory potentials in the retina: what do they reveal. Prog Retin Eye Res 17:485-521.

28. Kenyon GT, Moore B, Jeffs J, Denning KS, Stephens GJ, Travis BJ, George JS, Theiler J, Marshak DW (2003) A model of high-frequency oscillatory potentials in retinal ganglion cells. Vis Neurosci 20:465-480.

29. Kenyon GT, Travis BJ, Theiler J, George JS, Stephens GJ, Marshak DW (2004) Stimulus-specific oscillations in a retinal model. IEEE Trans Neural Netw 15:1083-1091. 